\title{
Simulation regarding the forward part of underwater remotely operated vehicle designed for research in maritime and river districts
}

\author{
Andra-Teodora Nedelcu ${ }^{1 *}$, Nicolae Buzbuchi ${ }^{2}$, Liviu-Constantin $\mathrm{Stan}^{2}$ and Mihail Lucian \\ Dumitrache $^{2}$ \\ ${ }^{1}$ Mircea cel Bătrân Naval Academy, Faculty of Navigation and Naval Transport, 1 Fulgerului Street, \\ 900218 Constanta, Romania \\ ${ }^{2}$ Constanta Maritime University, Faculty of Navigation and Naval Transport, 104 Mircea cel Batran \\ Street, 900663 Constanta, Romania
}

\begin{abstract}
Over the last few decades, underwater mechanical system became a necessity for ocean research and exploration. The necessity of this type of equipment provides a new type of marine platforms in different areas of oceanographic research. Until this moment, the underwater vehicles have developed in different shapes, sizes and means of propulsion. Take into consideration these characteristics, we could determinate the type and mission of the vehicle. In this paper we investigate the problem regarding the pressure exerted of remotely operated vehicle. The remotely operated vehicle is designed in order to achieve different mission regarding the surveillance in lakes, harbors and maritime environment. This mission can take place through a remote control system placed onshore or on seaborne platforms. To improve the endurance of remotely operated vehicle, we performed a simulation study during which we compare the drag forces acts on the underwater vehicle according to its shape. Using SolidWorks program to create the body simulation vehicle and Ansys CFX to realise the simulation, this paper present the result simulated for the forward part of the vehicle.
\end{abstract}

\section{Introduction}

A Remotely Operated Vehicle (ROV) provide an important role regarding collecting various oceano-graphic information, defense missions or applications, petrol, gas and different mineral exploration programs or biology studies. Using various integrated equipments and sensors, the vehicle could assure different assignments such as sea bottom topography, submarine detection or research the behavior of marine wildlife. Due to the fact that the remote control system is placed onshore or on seaborne platforms, an important characteristic represent the good endurance to complete different missions. Optimizing the vehicle shape it is necessary for energy utilization and endurance.

${ }^{*}$ Corresponding author: francu andrat@yahoo.com 
There was reported many studies [1]-[3] regarding the viscous fluid flow around different body shapes, for example cylinder, sphere or spheroid. The Navier-Stokes Equations explain explains and presents this behaviour regarding the viscous of fluid flow, which represent a particular forms of Newton's Laws of Motion. For complete solution of equations, it is necessary to be solved numerically in collaboration with a computer. To reach a valid solution, the continuous flow domain must be converted in a discrete domain, represented by a numerical mesh. Using numerical discretisation scheme, we perform algebraic equation. Using a computer, the coupled algebraic equations from numerical discretisation scheme are then solved and determinate discrete values of velocity and pressure at mesh nodes along the surface body or in different points. All this process represented by the theoretical, numerical and computational techniques gather in Computational Fluid Dynamics (CFD) simulation. Also, the CFD simulation could be turbulence, thus being used for dispersion, mixing and vibrations. The turbulence play an important role regarding the momentum distribution.

There are many studies effectuated in which the authors uses CTD simulation in different submersible and underwater vehicle projects reducing costs and save a lot of time compared with experimental tests. For example in his article, Barros et al.[4] compared the analytical results obtained with numerical results. As a conclusion, the CFD method is very usefully because help to realise an economically evaluating regarding the manoeuvrability of an underwater vehicle.

\section{Methodology}

\subsection{The geometry of body vehicle}

The shape of an underwater vehicle is similar to that of a torpedo type (in other words, in a form of cylindrical body with the front part rounded). At the first design stage using SolidWorks program, the hull can be divided into four sections, namely the nose (the forward part of vehicle), middle (the part where are located the sensors and sonars equipment), tail (the after part) and the propulsion used to propel the vehicle. To model the profile of the front and after part (or we can named the bow and stern- making an analogy to the ship parts) of the vehicle body it was used Myring Equations [5]. The Myring hull profile Equations describes curves information regarding the bow and stern of the vehicle body which in the end generate the smallest possible drag coefficient along the body vehicle.

The bow is described using a modified semielliptical radius distribution described as equation (1):

$$
r(x)=\frac{1}{2} d\left[1-\left(\frac{x-a}{a}\right)^{2}\right]^{\frac{1}{n}}
$$

Furthermore, the stern is defined using a cubic relationship described as equation (2):

$$
r(x)=\frac{1}{2} d-\left(\frac{3 d}{2 c^{2}}-\frac{\tan \theta}{c}\right)(x-a-b)^{2}+\left(\frac{d}{c^{3}}-\frac{\tan \theta}{c^{2}}\right)(x-a-b)^{3}
$$

Where $x$ represent the axial distance to the bow, $a, b, c$ represent the lengths of the bow, middle and stern. The parameter $d$ represent the middle hull diameter, $n$ represent the index of the stern shape, and $\theta$ the stern semi-angle. All there parameters are geometric, 
expect of the parametern. In Figure 1 are shown the schematic figure of an underwater vehicle hull and also the geometric parameters of Myring Equations.

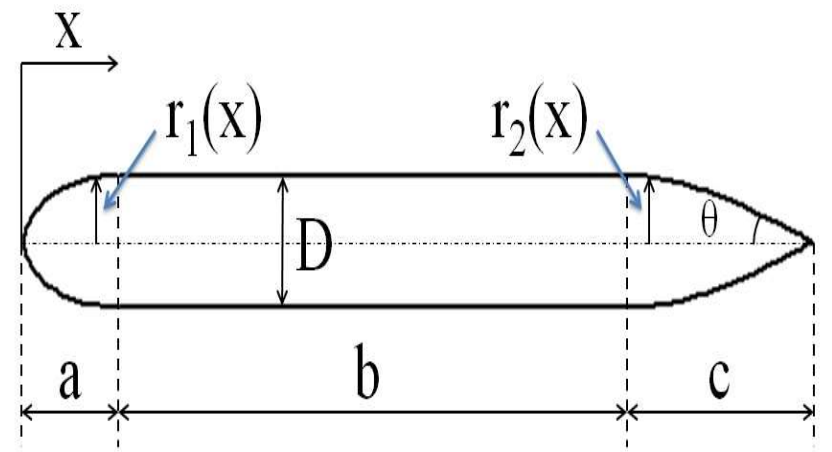

Fig. 1. Schematic figure represent the underwater vehicle hull and geometric parameters of Myring Equations.

\subsection{Hull design}

The concept design of the underwater vehicle is shown in Figure 2. To realise the design was used Solid Works Program.

Fig. 2. The concept design using SolidWorks Program

The steps of design the vehicle hull was as follow:

1. On the first step, was created a Sketch with vehicle body dimensions;

2. After, we doubling this Sketch created on first step in the OY direction to generate the lower part of the body vehicle along the OX axis (Figure 3); 


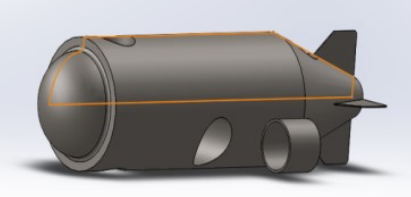

Fig. 3. Doubling the Sketch in the OY direction

3. Using the Mirror function, we create create mirrors propulsion on both sides of the vehicle; (Figure 4)

Fig. 4.Mirror propulsion on both sides

4. To realize the tail of the vehicle body, we create a new plan;

5. To create the from the tail of the vehicle was used The CirPattern function;

6. To fill the vehicle on both sides of the axle was used Revolve function;

7. Using Cut-Extrude function was create holes on the body of the vehicle;(Figure 5). 


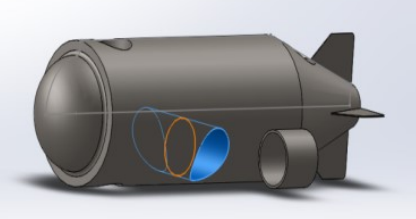

Fig. 5. The holes crated on the body vehicle

\subsection{Numerical basis and turbulence model}

To realize the simulation was used Ansys CFD software [7] solver.

The CFD simulation process involves three main stages:

- Pre-processing

- Solution

- Post-processing

The methodology for performing a CFD simulation includes several stages as follows:

- modelling, which involves creating the geometry step by step. First, points are created, joined by curves, then surfaces are created, and finally the body and field required to perform the analysis are generated.

- meshing (creation of the node network) also bears the name and discretization and represents a discrete representation of the geometry for each individual region which is then used to generate the volume of the node network. The meshing is made up of triangular and vertical faces.

The domain called "network" or "mesh is then discretized into a finite set of control volumes or cells. General equations of conservation of mass and momentum are discretized into algebraic equations. All equations listed are solved to create the flow field:

- calculation method;

- defining the boundary conditions;

- solution;

- post processing, which involves analyzing the results in one of two methods, graphically or alpha-numerically.

The graph shows contours, vectors, graphs, iso-surfaces, power lines and animations. Numerical are shown the integral values, calculations on the resistance to advancement, the distribution of pressure on the body surface compared to the experimental data.

The underwater vehicle is moving into an environment known as domain. In order to realise the simulation, a field considered wide enough was created to prevent the domain boundaries from affecting the flow along all the body vehicle. The dimensions of the computational domain (general parameters as length, width, depth) along the underwater vehicle are shown in Figure 6. 
Fig. 6. The domain

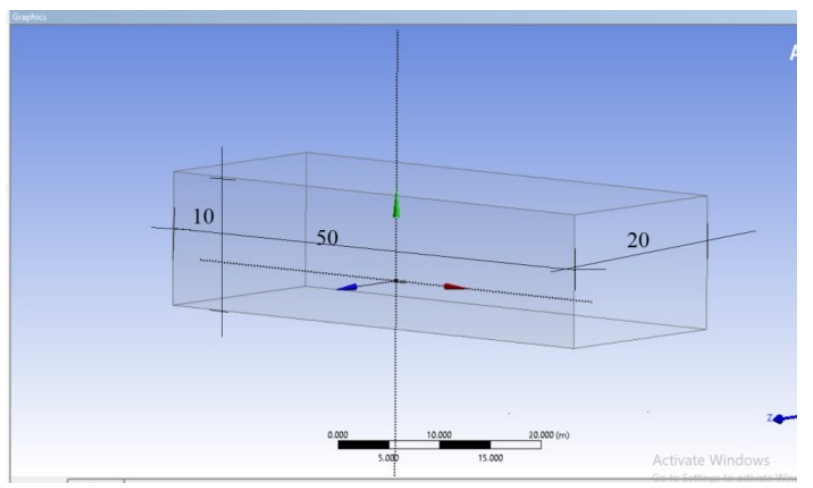

For numerical determinations we consider the domain as a parallelepiped that encloses the body of the vehicle.

\subsubsection{Generation of meshing}

In order to numerically analyze the flow, the physical domain of fluid is discretized into network cells.[8] Flow variables are associated with each cell (element) in the network. This model uses the second-order finite element method. Compared to first-order discretization, it offers greater network convergence and greater discretization accuracy. Figure 7 shows the obtained cell network for the fluid domain in one of the studied cases.

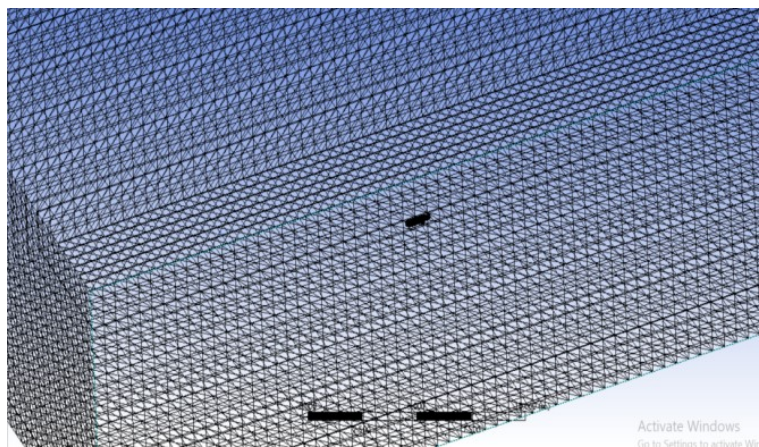

(a) General view

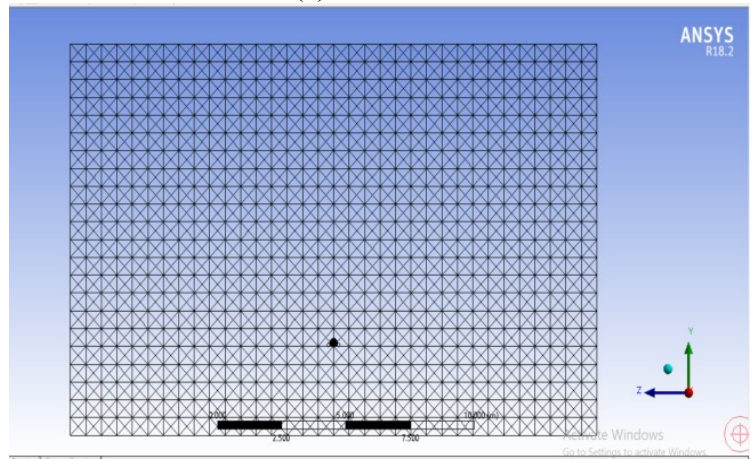

(b) Bow zone 


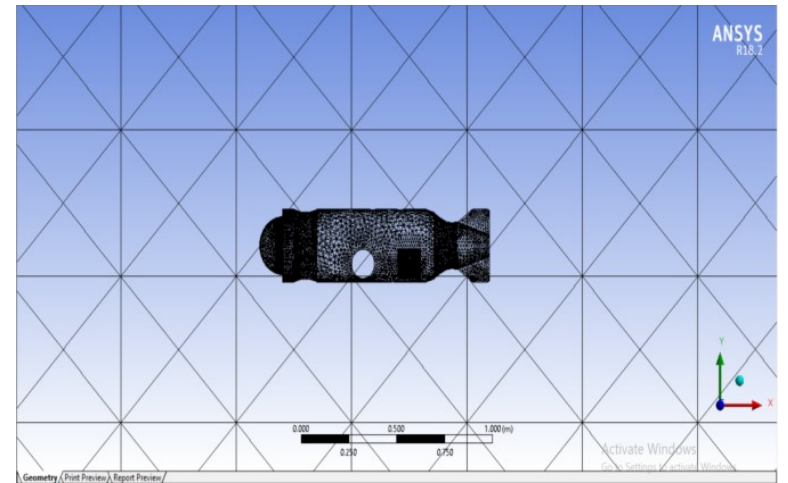

(c) Layers of cells along the body of the vehicle

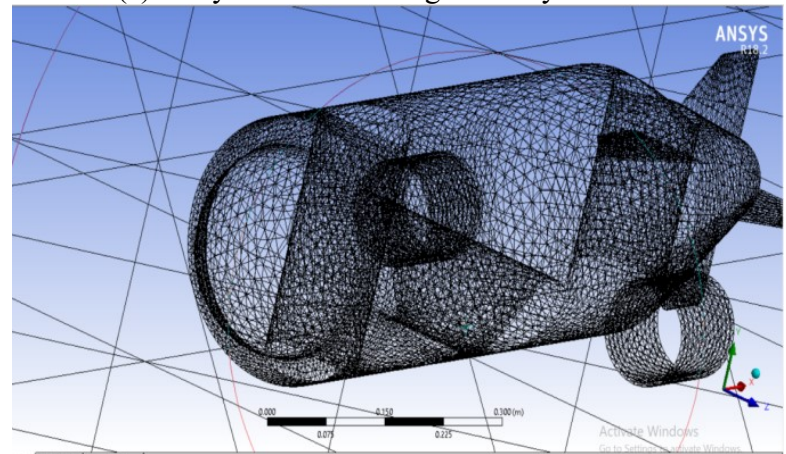

(d) Bow section of the vehicle

Fig. 7. Meshing

The conditions imposed on the mesh are: size function - curvature, maxim face size $0.605520 \mathrm{~m}$, mesh deseature size $3.0276 \mathrm{e}-003 \mathrm{~m}$, transition growth rate 1.1280 , bounding box $56.7370 \mathrm{~m}$ and minimum edge $8.0131 \mathrm{e}-006 \mathrm{~m}$.

To generate the phenomenon of flow during the simulations, the density of the node network was more focused in different regions of the domain. Regarding this, a fine mesh was used along the surface of the all body vehicle to resolve the equation of flow in the boundary layer.

The Skewness and Orthogonal quality functions from Mesh Statistics - Mesh Metric were used to determinate the quality of the generated network, these being the most important methods for determining the quality of the network.

The results show in final 163385 knots and 912137 elements was formed in mesh.

\subsubsection{Convergence criterion}

To decide if a solution has reached the desired level of convergence it is useful to monitor the residuals of the flow variables for each iteration. The residuals represent a measure of the imbalance between the left and right sides of a discretized transport equation. They are the most important measures of convergence, because they are directly related to the correct solution of the equations.

The CFX solver uses standardized residuals to determine convergence. Standard residuals are used to automatically stop when the solver reached a specified level. To observe when convergence is achieved, the user must select the residual type, MAX (maximum) or RMS (root mean square) and specify a target value. In the CFD simulations, 
the RMS was used residuals with a target value of 10-5, which represents according the program license a good convergence and is considered sufficient for the most part of engineering applications.

\subsubsection{Boundary conditions}

The condition at the output limit used was the pressure outlet, which sets the value of static pressure at the output limit, being the most suitable for CFD marine simulations. It can be assumed that the output pressure is equal to the hydrostatic pressure, if the location of the output limit is made far enough from the target object of the turbulence simulation in the flow field.

We considered the vehicle positioned at a single depth and moving with constant speed. Furthermore, we add the following assumptions and simplifications:

- consider the incompressible fluid be homogeneous flow;

- the vehicle is moving linear with constant speed;

- free surface;

- during the simulation, we not perform a dynamic mesh;

- the geometrical design of the vehicle was done on a real scale according to reality;

- fluid flow was realize in a parallelepiped area around the all body of the vehicle.

The condition imposed for the domain entry limit was of the inlet type, and the fluid velocity corresponded to the relative velocity of the body vehicle achieved by summing the velocity of the body vehicle and the velocity of the water current for each simulation. The simulation speed was $10 \mathrm{~m} / \mathrm{s}$. The flow regime was defined as subsonic, the mass and moment having normal speed values. The turbulent intensity was defined as average and the Eddy viscosity coefficient was taken into account.

The condition imposed at the output limit of the domain was of the output type, with the option of the subsonic flow regime, the mass and the moment at normal speed, and the corresponding output speed having the same value as the input.

The condition imposed on the bottom of the domain (bottom) was of the no slip wall type. Also, the roughness of the bottom of the domain was defined as a smooth wall.

The properties of the water used in the simulations are density $1000 \mathrm{~kg} / \mathrm{m} 3$, the model of heat transfer being isothermal, the fluid temperature of $25^{\circ} \mathrm{C}$. The model used for turbulence is SST (hear-stress transport)[6].

\subsection{Model the flow around the body of the vehicle}

The vehicle body position varies along the OY direction, the simulations was performed for different positions of the body vehicle.

The moving of the vehicle was considered stationary and subsonic. For modeling, wrte not taken into account the heat exchange and weight forces. We consider the wall roughness to be $5 \mu \mathrm{m}$.

During the simulations the vehicle speed and current acting along the body vehicle summed a total of $10 \mathrm{~m} / \mathrm{s}$, we consider the average temperature of water to be 25 degrees. The vehicle is positioned to a maximum depth of 7 meters.

Figure 8 present the total pressure along the vehicle body. The simulation show a maximum value of pressure of $50891.5 \mathrm{~Pa}$, reached in the front part of the vehicle , according to the legend where the maximum value of pressure is represented by colour red. 
The minimum reached is represented by a value of $-92984.5 \mathrm{~Pa}$. The minimum value is located in the immediate vicinity of the vehicle's hull, besides the front part of vehicle and is represented in figure 8 using blue color.

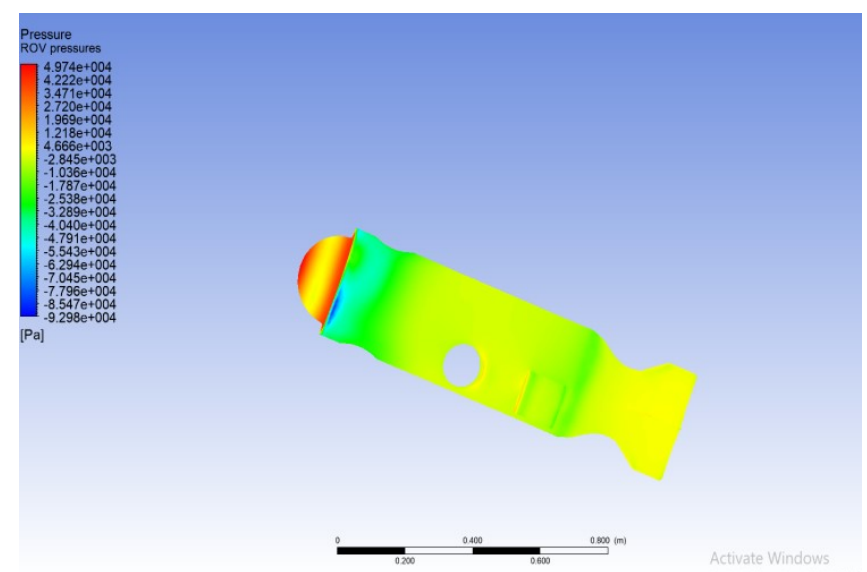

Fig. 8. Simulation regarding the pressure along the body vehicle

In Figure 9 and Figure 10 are presented the streamlines, pressure gradient lines, respectively the velocity that appear along the vehicle body.

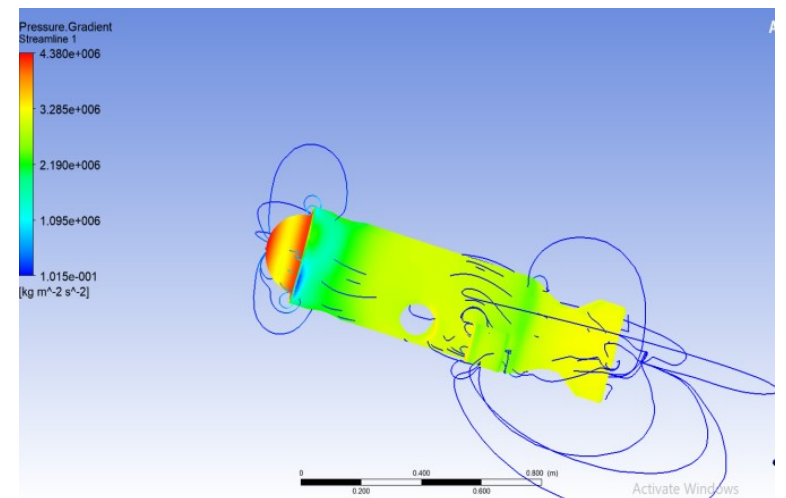

Fig. 9. Streamlines along the vehicle body - present pressure gradient

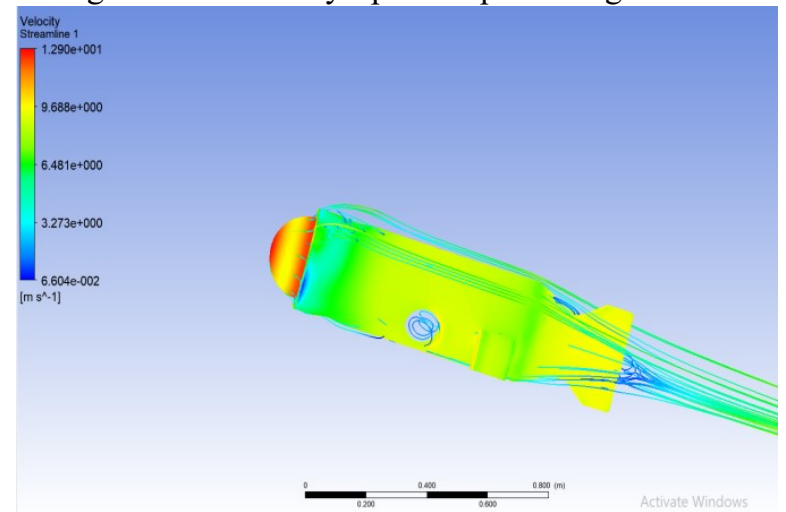

Fig. 10. Streamlines along the vehicle body - present velocity 
In Figure 11, is presented the pressure distribution along the transverse surface of the body vehicle. Take into consideration the simulation, we notice a zone of maximum of 50891.4 Pa pressure in the front part of vehicle body marked with red colour, then a minimum area in the immediate vicinity of the front part of body vehicle. The average area, marked with green colour, is located near the prow, next to the propellers vertically and towards the stern of the vehicle.

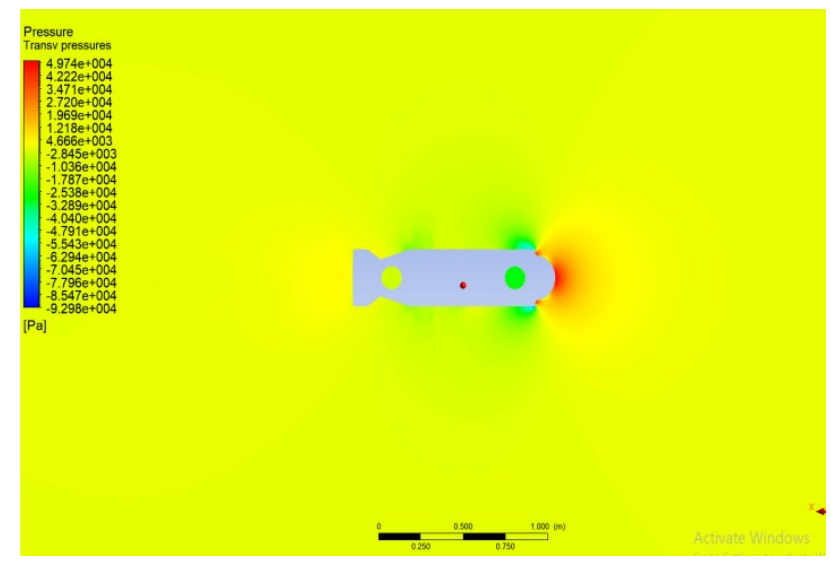

Fig. 11. The transverse surface of the body vehicle - pressure distribution

\section{Conclusion}

This paper presents a hydrodynamic single-pass flow distribution regarding the pressure distribution around of an underwater vehicle body.

In the future, the simulated results will be verified using the experimental tests in tank and after that in sea water. We consider that future works could improve and establish the accuracy of the CFD results comparing the numerical simulation with the underwater vehicle using a real-time adaptive identification approach in sea trial.

Vehicles can determine areas of interest for operation. The underwater vehicle has improved our ability and perspective to research the sea-floor, providing higher resolution of seafloor mapping data.

\section{References}

1. Proudman, I. and Pearson, J.R.A. (1957) Expansions at Small Reynolds Numbers for the Flow Past a Sphere and a Circular Cylinder. Journal of Fluid Mechanics, 2, 237262. http://dx.doi.org/10.1017/S0022112057000105

2. Masliyah, J.H. and Epstein, N. (1971) Steady Symmetric Flow Past Elliptical Cylinder. Industrial \& Engineering Chemistry Fundaments, 10, 293-299. http://dx.doi.org/10.1021/i160038a017

3. Rosenfeld, M., Wolfshtein, M. and Israeli, M. (1992) A Numerical Study of the Laminar Incompressible Flow Over a Prolate Spheroid at $10^{\circ}$ Incidence. International Journal for Numerical Methods in Fluids, 15, 147-173. http://dx.doi.org/10.1002/fld.1650150203 
4. Barros, E.A., Dantas, J.L.D., Pascoal, A.M. and Sá, E. (2008) Investigation of Normal Force and Moment Coefficients for an AUV at Nonlinear Angle of Attack and Sideslip Range. IEEE Journal of Oceanic Engineering, 33, 538-549. http://dx.doi.org/10.1109/JOE.2008.2004761

5. Myring, D.F. (1976) A Theoretical Study of Body Drag in Subcritical Axisymmetric Flow. The Aeronautical Quartely, 27, 186-194.

6. Menter, F. R. (August 1994). "Two-Equation Eddy-Viscosity Turbulence Models for Engineering Applications". AIAA Journal. 32 (8): 1598-1605. Bib code:1994AIAAJ .32.1598M. doi:10.2514/3.12149.

7. ANSYS CFX- Solver Theory, ANSYS, Inc., Canonsburg.

8. Juong, T., Sammut, K., He, F. and Lee, S.K. (2009) A Study on the Design Optimization of an AUV by Using Computational Fluid Dynamic Analysis. Proceedings of the 19th International Offshore and Polar Engineering Conference, Osaka, 21-26 June 2009, 696-702. 